\title{
Design and Implementation of Flow Meter Maintenance Robot for Oil \& Gas Industries
}

\author{
Khalifa Al Zaabi ${ }^{1}$, Javeed Hussain ${ }^{2}$ \\ ${ }^{1}$ Department of Electronics and Telecommunication Engineering \\ ${ }^{2}$ Senior Lecturer, Department of Electronics and Telecommunication Engineering \\ Global College of Engineering and Technology, Muscat, Oman
}

\begin{abstract}
Robotics technology is that has been implemented to demonstrate the human ideas into life reality and used to solve the difficulty of human access. In this paper a robot is designed to clean confined places such as pipe lines. The use of robots is more common today than ever before. Robotics used in precision cutting, electrical, danger places, welding, medical failed and other uses. Several designs of In-pipe inspection robots have been proposed in the literature to solve the problems related with inspection of these complicated internal geometries. Designing of an in-pipe inspection robot is a difficult task, especially when its move through critical devices inside the pipe such as magnetic flow meter. In this paper, designed a robot that can enter a pipe line that equipped with magnetic flow meter. This idea is appeared while working in oil field company that has some units equipped with 12 " magnetic flow meter installed in a pipe line. The flow meter used to measure the flow of conductive fluid such as water, gel or other water base chemical. It became contaminated after certain months of used and start to show fluctuation in reading. This robot is equipped with camera and I implemented using android software APK to control robot using Bluetooth module.
\end{abstract}

\section{INTRODUCTION}

Robotics is one of the fastest growing engineering fields of today. Robots are designed to eliminate the human factor from intensive or dangerous work and also, to reach to an inaccessible environment. The robots use is more common today and it is no longer exclusively used by the large production industries. The pipes inspection may be relevant for improving security and efficiency in industrial area. These specific operations as inspection, maintenance, cleaning etc. are expensive, the robot's application appears to be one of the most attractive solutions. The pipelines are the major tools for the transportation of effluent water, drinkable water, gas and fuel oils. A lot of troubles caused by piping networks aging, cracks, corrosion, and mechanical damages are possible. So, activities for inspection, maintenance and repair are strongly required. The robots with a flexible structure are adaptable to the environment, especially to the pipe diameter, maneuverability and capability to operate under hostile conditions.

The aim of this work is to design and fabricate 12' Pipeline Robot for investigation, cleaning and monitoring of pipe line that installed with magnetic flow meter which used to measure the flow rate of a conductive fluid. The idea appears while I was working with an oil field service company and recognized an issue with magnetic flow meter showed wrong flow rate. Trouble shooting carried out and found that the electrode of the flow meter become contaminated and affect the amount of correct induced voltages received by the electrode. Figure (1) show a unit with two magnetic flow meters installed in suction line, the flow meter can be removed from the pipe for cleaning, but it's too heavy to do so in remote location and too risky to do it without overhead crane. For this reason, they need to send the unit to base location which is $450 \mathrm{KM}$ far, for at least five days.
The idea of making a magnetic flow meter cleaning robot will reduce the inspection time, cost of man power and minimize the potential risk. There are many robots with different features. In this project, the design of robot is undertaken with an extra feature work with mobile remote-control app for distance and fitted with video camera to take photographs inside the pipeline to identify the faults of pipe or flow meter.

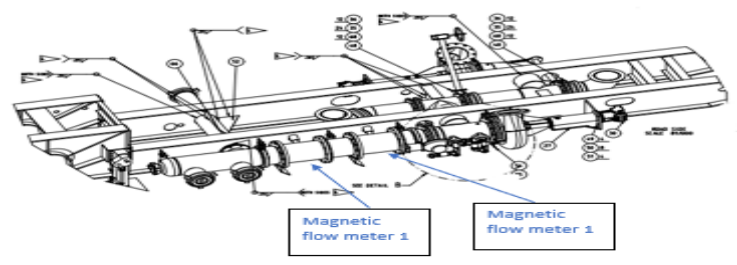

Figure (1) Magnetic flow meter in a unit 12" pipe [1]

The pipe of the main suction flowmeter was found dirty Figure (2), causing the flowmeter reading to be inaccurate. By cleaning the magnetic flow meter electrode area, the flow rate reading will become more accurate and has no fluctuation.

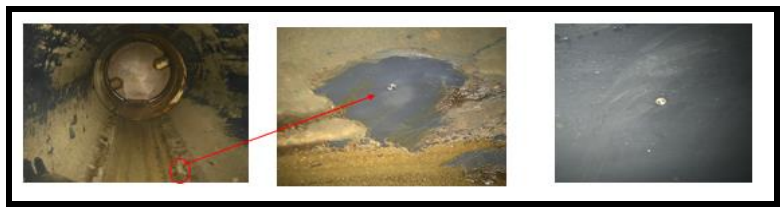

Figure (2) Gel mixer unit showing dirty pipe

\section{LITERATURE SURVEY}

In the following items are showing the pipe robots, developed by different research centers around the World such as the Hiroshi \& Yoneda laboratory of Tokyo Institute of Technology, the laboratory of active structures of Brussels University, the Robotics laboratory research center of Petrobras, Mobile robotics laboratory University of Michigan, CTIT University of Twente, Biomimetic Intelligent Mechatronics Lab Ritsumeikan, MIT, Robotic institute of Carnegie Mellon and companies how Tuboscope, Roboprobe, Inuktum, Inspection Probe and

Toshiba among others [2].

\section{a. General pipe robots}

Research laboratories and industries around the world made big efforts to develop different structures that can move into pipes at the figure (3) are showed some of in pipe robots organized by its locomotion mechanism. 


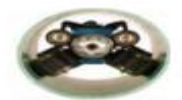

(a)

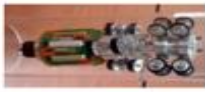

(d)

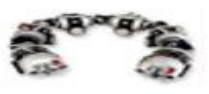

(g)

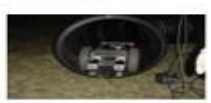

(j)

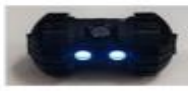

(m) (b)

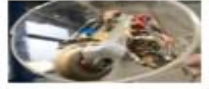

(e)

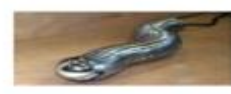

(h)

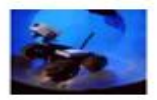

(k)

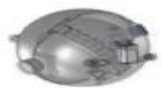

(n)

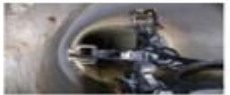

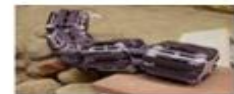

(c)

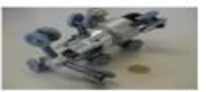

(f)

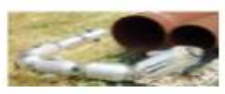

(i)

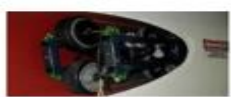

(1)

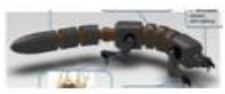

(o)
Figure (3) General pipe robots [2].

The figure (3) presents the in pipe robots, the present robot label $(\mathrm{a}-\mathrm{c})$ are Crawler type robots, $(\mathrm{d}-\mathrm{f})$ are robots that uses the pipe perimeter how path to travel, the $(g-i)$ are Snake type, $(j-1)$ are traditional robot of four wheels, (m) two wheels, (n) ball type, (o) is a robot with legs. All they were focused in solve the problem of locomotion to travel into different pipes types, these robots can carry a camera and obtain images inside the pipes, have electrical motors and corresponds to prototypes, only the reference (a) is currently a commercial robot [2].

\section{b. Robots to pipeline construction and maintenance}

To aid the pipeline construction and maintenance were developed special robotics devices they are applied to milling, grinding, polishing, welding and piping unlocking. They are presented at figure (4).

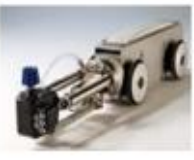

(a)

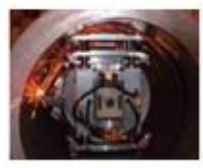

(d)

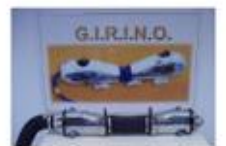

(g)

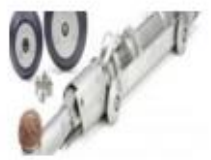

(j)

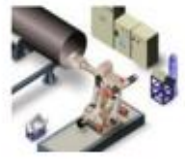

(b)

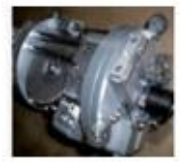

(e)

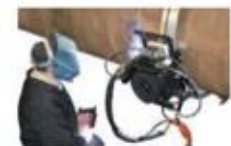

(h)

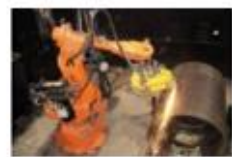

(k)

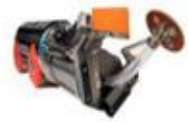

(c)

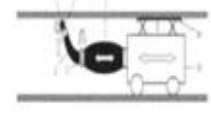

(f)

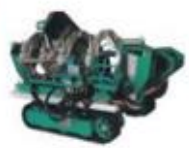

(i)

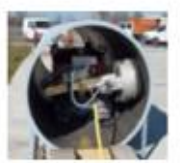

(I)
The figure (4) presented the robots to construction and maintenance of pipelines, the robot of label (a) is used to milling, the (b) mark and prepare the pipe to welding, (c) fix the holes, $(\mathrm{d}, \mathrm{e}, \mathrm{j}, \mathrm{k})$ to polished the pipeline wall, $(\mathrm{g})$ to unlock the pipes, $(\mathrm{h}, \mathrm{i})$ are welding robots to pipes and (l) to cleaning the pipeline without flux [2].

\section{PROJECT ROBOT DESIGN}

The purpose of this project is to design a 12" pipeline robot that used to clean the electrode of a magnetic flow meter figure (5) which used in some oil field units to measure a conductive fluid flow rate.

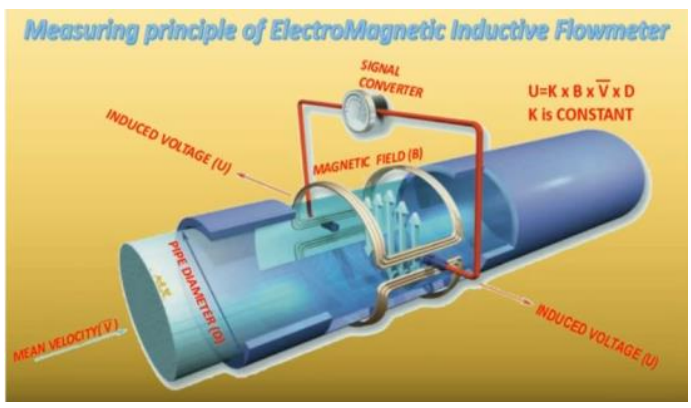

Figure (5) magnetic flow meter [3]

Also design an APK mobile software to connect the robot to mobile phone or use another remote-control panel via Bluetooth. More ever, use mobile wireless communication to control $2 \mathrm{MP}$ camera, which will give a good vision of pipe internal.

The flowmeter itself must have an insulating lining, which can be made from rubber, Teflon, ceramic, or other materials capable of standing up to erosive or corrosive media [3]. The electrode of the flow meter that used to received induced voltages while conductive flow occur is become contaminated which affect the reading of flow rate and keep it fluctuating.

\subsection{Design 12" cleaning robot:}

Designing of an in-pipe inspection robot (IPIR) is a difficult task and hence the designer must take care of the design issues like Mobility, Steer ability, Turning radius, Size and shape adaptability. This proposed model is a crawler wheel type In-pipe inspection robot. It is able to move through horizontal pipes and it can easily pass through elbow of a 12 " pipe line. This model robot figure (6) comprises of two crawler modules- rotor, cleaning arm, camera arm, microcontroller and control unit. The crawler module has two motors that rubber drive chain wheels to control robot maneuver. Cleaning arm has two axes servo motors to move the arm in 360 degree to cross pipe wall as well 180 degree of literal move. The camera arm is used to control the camera direction. ATMEGA328 microcontroller is used to control the robot maneuver, cleaning arm and camera arm movement according to the controller selection. Mobile remote wireless control unit is used to control the robot from distance and make sure it's in safe route and don't damage the flow meter pipe wall.

Figure (4) Robots to pipeline construction and maintenance[2] 


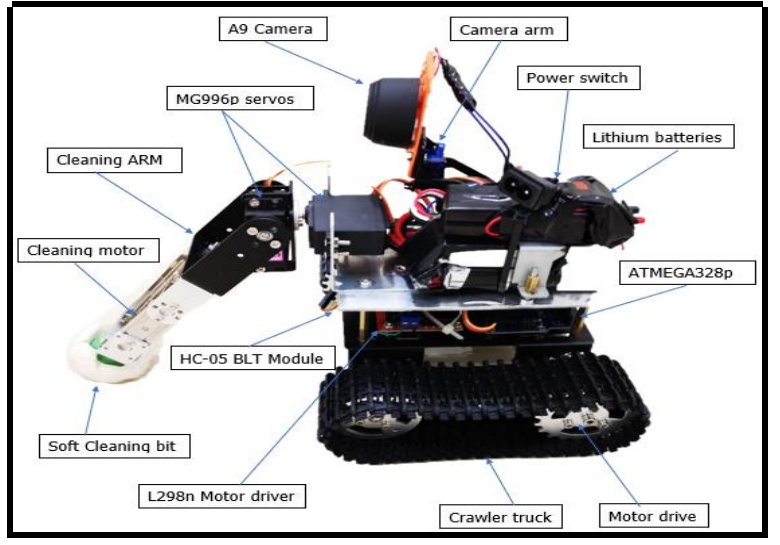

Figure (6) 12" cleaning robot parts description

\section{1. 1 flow chart:}

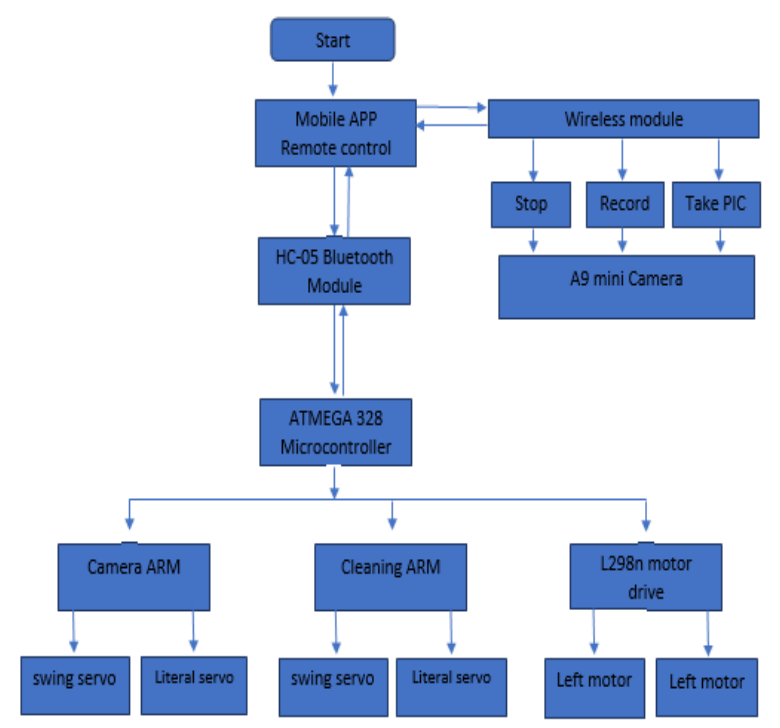

\subsubsection{Rotor module:}

Similarly, to most of the analyzed robots' structures, it was decided to utilize two crawler tracks. That configuration ensures proper robot stability and maneuverability. The main body of the cleaning robot is consisting of three main items, which is $6-12 \mathrm{v}$ motors, crawler truck and metal chassis to support the motors and arms that used for cleaning and camera direction control.

The crawling wheels is made of rubber material to ensure it will not scratch the pipe or the electrode of flowmeter. It supported by metal chassis that handle wheel motors and for more stability. The dimension of the robot chassis with crawling wheels is $19 \mathrm{~cm}, 17 \mathrm{~cm}$ and $5.7 \mathrm{~cm}(\mathrm{~L}, \mathrm{~W}, \mathrm{H})$ figure (7). which make the robot is shoutable to enter a 12 " pipe and move freely to clean the electrodes of the flowmeter.

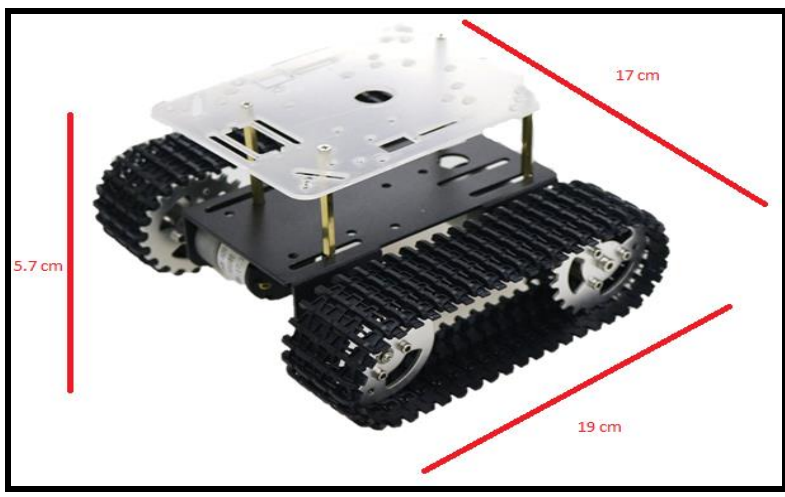

Figure (7) rotor driver dimensions.

For this project I have select a Rubber crawler track wheel, the reason to select the rubber truck is to have a good grip maneuver inside the pipe and its un-scratchable material. For better grip to the pipe wall we need to install a 40-angle motor driver bracket which will make the crawler rubber wheel is if fully driven on pipe wall figure (8).

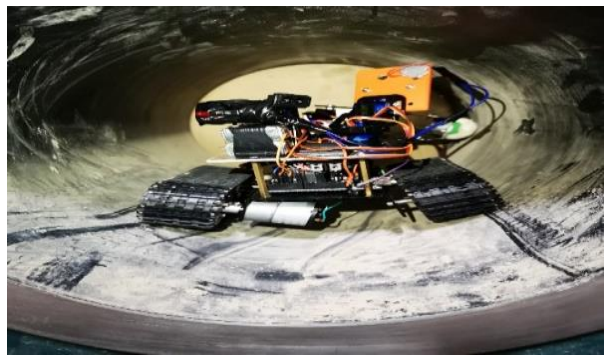

Figure (8) 40 angle motor driver bracket

\subsubsection{Cleaning arm:}

The robot is designed to hold a cleaning arm figure (9) that has two axes. The length of the arm is adjustable, which can be setup according to the pipe size. Its maximum length is $15 \mathrm{~cm}$. Its equipped with two MG996 servo motors to move the arm in two axes with 360 degree range for one servo that used to rotate the ARM around the pipe wall and 180 degree of movement for the other servo. Also, one dome shape soft material that secured to 5 vdc motor, which used to clean the flowmeter electrode without scratch it.

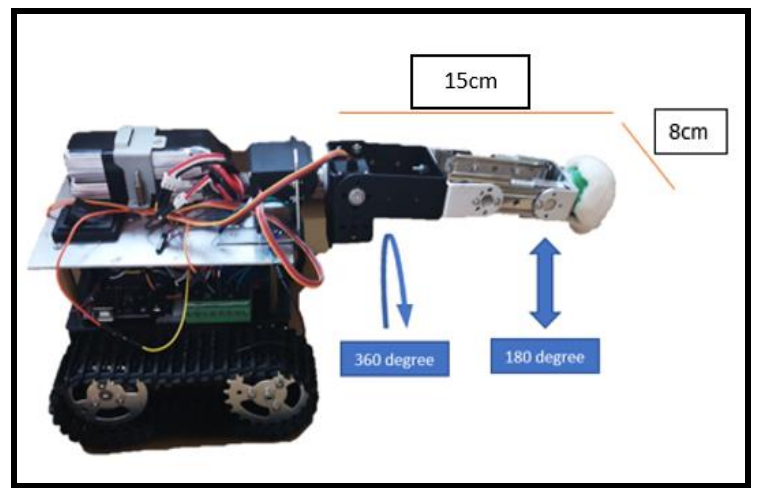

Figure (9) Cleaning arm dimensions

The High-Torque MG996R Digital Servo features metal gearing resulting in extra high $10 \mathrm{~kg}$ stalling torque in a tiny package. It features upgraded shock-proofing and a edesigned PCB and IC control system that make it much more accurate than its predecessor MG995 [4]. 


\subsubsection{Camera arm:}

In this project I have decide to install a mini camera that is capable to send a good vision via robot control unit. A9 1080P HD mini camera is installed for this robot. Its provide 150 wide angle range and support night vision. It's used P2P wireless connection, or it can be connected through a router. The camera is installed in two axes arm figure (11) that equipped with two SG90 servo motors which can move 360 degree in each axis. Moreover, one $10 \mathrm{w}$ LED light is installed on top of camera to give required illumination for the camera view.

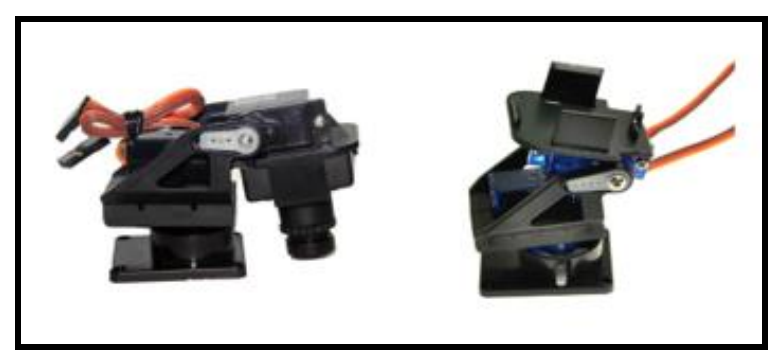

Figure (10) camera arm

\subsubsection{Robot ATMEGA 328P Microcontroller:}

To control the cleaning robot, we need a microcontroller that help the robot to be self-controlled without human touch. For this cleaning robot we are going to use Atmega 328P microcontroller. It's is high performance, low power controller from Microchip. ATMEGA328P is an 8-bit microcontroller based on AVR RISC architecture. It is the most popular of all AVR controllers as it is used in microcontroller boards.

\subsubsection{Robot App Control unit:}

A several ways can be used to control the robot through cable or wireless. It can be hand held control unit or using mobile phone or IPAD with implemented application. For this robot I have use mobile phone to communicate with the robot via Bluetooth module HC-05. It can control the robot up to $14 \mathrm{~m}$ in free space and 10 meters in confied space such as pipe line. At the same time it communicate by wireless to A9 1080P HD mini camera for faster picture transferring rather than Bluetooth camera module.

\subsubsection{Robot assembling:}

To assemble the robot, a certain aspect we need to think about it such as dimension, balancing, arms restriction, center of Geraghty, rotor tension and safe wiring looms route. Also, following the wiring in wiring diagram figure (11) is important.

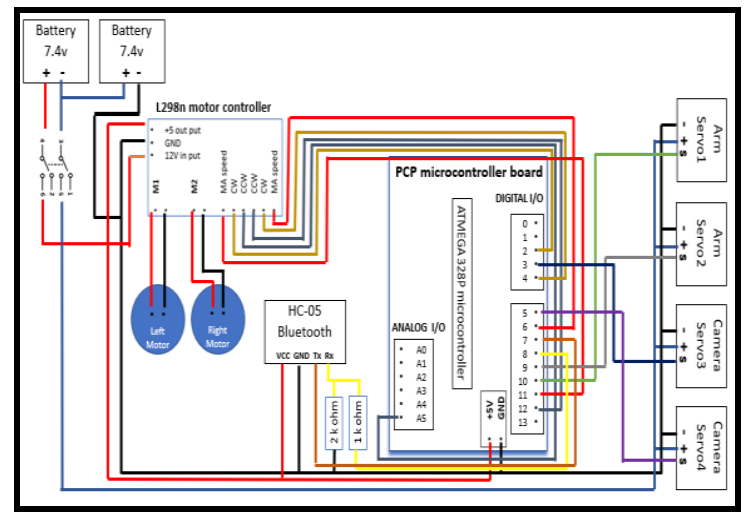

Figure (11) cleaning robot wiring diagram

Full functional test should be carried out after assembling the robot in figure (12). All robot section to be tested and certain adjustment to the c code of microcontroller to be changed according to the required actuator output. Also, using the c code we can limit the maximum and minimum edge limitation of the servo motors to work in safe area.

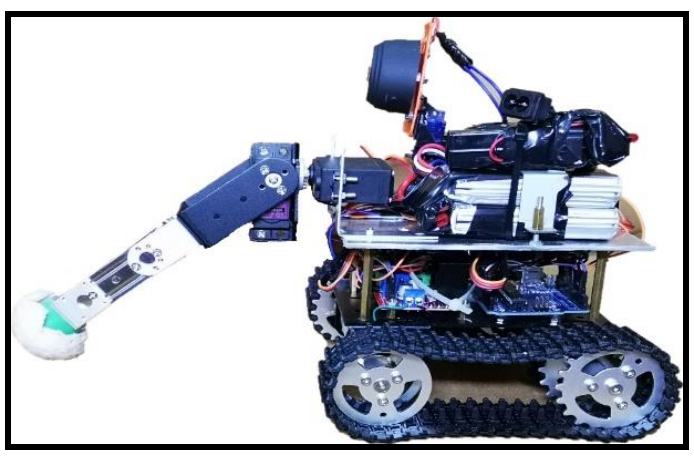

Figure (12) Assembling cleaning robot

\subsection{Implementing mobile APK:}

The quick and easy start and the equally quick and intuitive development of attractive and demanding apps are the declared aims of the visual development tool App Inventor. App Inventor is aimed at a far larger target group than ordinary development tools. With App Inventor, all users of Android smartphones now have the chance to peek behind the scenes at the colorful world of apps, then have a go themselves and express their creativity by designing their own apps. [5].

App Inventor is an educational learning tool provided by MIT that allows users to build Android apps without any knowledge of programming. As App Inventor gains opularity amongst educators and students around the world, it will become increasingly important to ensure that the tool offers its users the breadth and depth of app-development functionality they desire [6].

\subsection{Robot Test and mentoring:}

In order to integrate a component within a larger system, three major properties, the fitness, the correctness, and the robustness, must be tested. The fitness of a component for an application is in general treated as the compatibility of the provided interface of the component and the specification of the required interface of the application. The correctness of a component is its ability to return the correct output when provided with the correct input, while the robustness concerns the absence of a behavior possibly jeopardizing the rest of the system, especially under wrong input. When lot of components is present, integration testing became quite complex and one of the software development improvement steps pertains to testing process improvements which can hardly be done without test automation [7].

To make sure the project is working as been implemented for, each robot system hardware and software test to be carried out. Cleaning robot is divided to three main sections- rotor truck, cleaning ARM and camera ARM, which controlled by ATMEGA $328 \mathrm{p}$ microcontroller library codes. The microcontroller is connected to the mobile App control unit through Bluetooth module. 


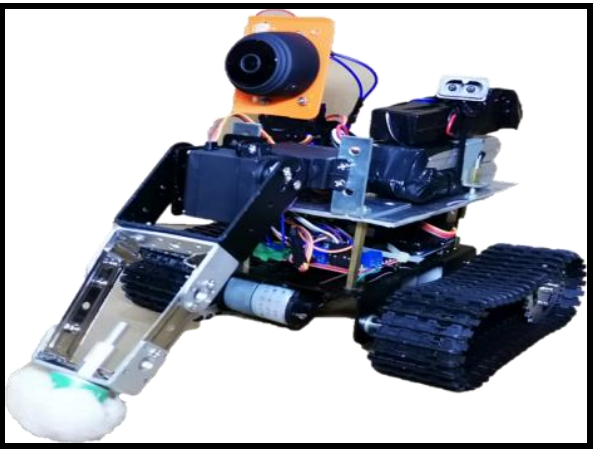

Figure (13) cleaning robot structure

\subsubsection{Mobile App control unit to microcontroller communication test:}

This test will make sure the communication between the control unit and the robot microcontroller is connected and it show a green connected word label in the control unit App. Before doing that, we need to make sure the script block code is mobile app is correct and no-fault errors figure (14). Also, the Bluetooth module is correctly wired to the microcontroller and the script code for Bluetooth module is downloaded correctly to the microcontroller figure (15).

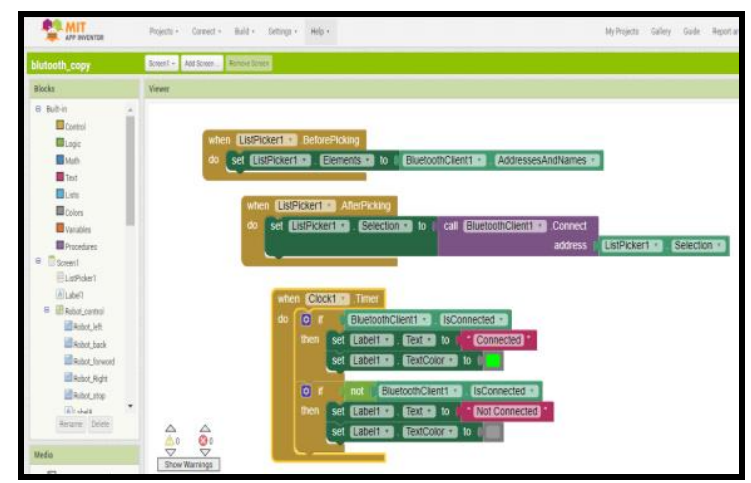

Figure (14) MIT inventor no fault show

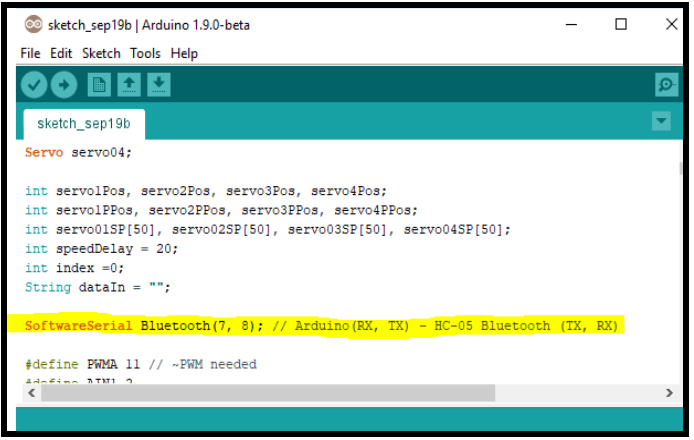

Figure (15) Bluetooth Serial Rx \& Tx pins

- Three steps to connect mobile control app to cleaning robot figure (16):

1- switch ON robot and Press Bluetooth icon on mobile control app.

2- Select Bluetooth module.

3- Check a green connected label word is showing in app main page.

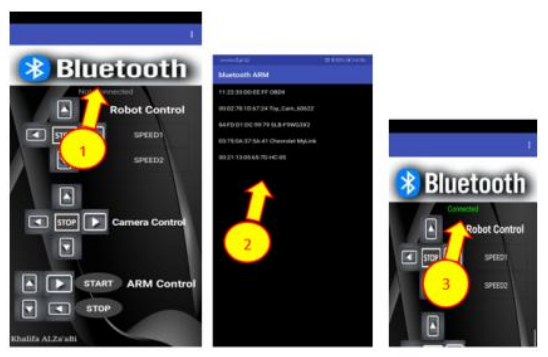

Figure (16) how to connect phone to HC-05 module

\subsubsection{Clearing ARM test:}

The c code is setup to drive the two axes drive servo MG996 to move the arm up, down and 360-degree round across wall pipe figure (17). Also, we can control the speed of the arm rotation speed by controlling the delay time of start moving from A to B point.

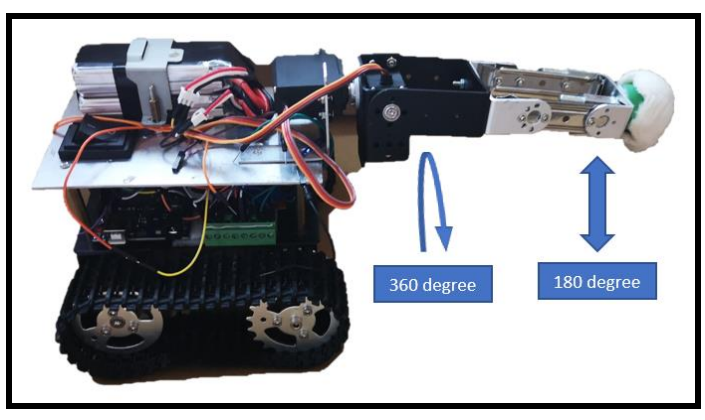

Figure (17) cleaning arm moving direction

ARM control section in mobile app figure (18) is used to move cleaning ARM up and down by press the arrows up or down. Also, it moves the ARM 360 degree across the pipe wall by pressing the left or right arrow.

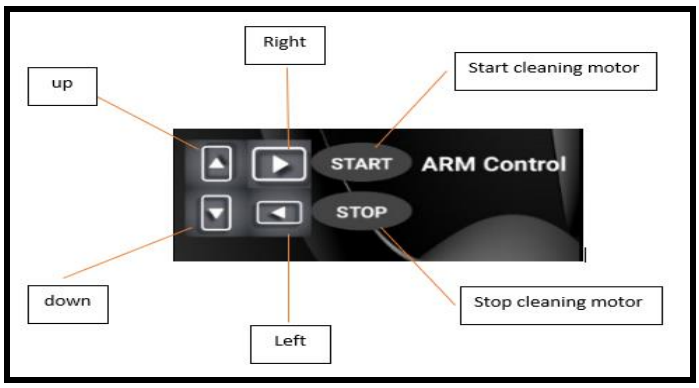

Figure (18) Mobile app ARM control section

Pressing any button of ARM control section is designed to send a command order through Bluetooth to microcontroller.

\section{CONCLUSION}

This robot is designed to replace the human from cleaning 12 " magnetic flow meter which installed in some units' pipes and needs frequently clean. The designed and fabricated of magnetic flow meter cleaning robot can move forward straight, backward, right direction and left direction and has a wireless camera to monitor the pipeline for contamination the affect the flow meter reading. Also, desired mobile application has been implemented to communicate with the cleaning robot by Bluetooth and make it easy for the operate to move the robot inside the pipe line to clean electrode of the magnetic flow meter. 


\section{REFERENCES}

[1] Katherine Palazzo, 2014, SBF-216 Slurry PCM manual [Stumberger]

[2] John Archila and Marcelo Becker, (2013). Study of Robots to Pipelines, Mathematical Models and Simulation. [IEEE Latin American Robotics Symposium]

[3] Yokogawa Electric Corporation, The Excitation Frequency of Magnetic Flowmeters https://www.yokogawa.com/library/resources/mediapublications/the-excitation-frequency-of-magnetic-flowmeters

[4] Handson Technology, user guide, MG996R Metal Gear Servo Motor https://www.handsontec.com/dataspecs/motor_fan/MG996R.pdf[access online]

[5] Jorg H. Kloss, 2012. Android Apps with App Inventor [ Addison-wesley]

[6] Anshul Bhagi,2011. Android Game Development with AppInventor [Massachusetts Institute of Technology]

[7] Stanislav Stresnjak, Zeljko Hocenski, 2015. Usage of Robot Framework in Automation of Functional Test Regression. [University Josip Juraj Strossmayer in Osijek]

[8] Gaddis \& Halsey, 2015. Starting out with App Inventor for Android. [www.pearsonglobaleditions.com]

[9] Handson Technology, user guide, L298N Dual H-Bridge Motor Driver. http://www.handsontec.com/dataspecs/L298N\%20Motor\%20Driver.pdf [access online]

[10] Media wilpe, 2019, 1080P HD SPY WIFI CAMERA (user manual) https://media.wilpe.com/pdf/17732-sw04-wifi-spy-clock-billingualmanual.pdf [access online]

[11] MIT App Inventor 2, Pearson Education, Inc http://ai2.appinventor.mit.edu/

[12] Mrs. Anisha Cotta, Miss. Naik Trupti Devidas, Miss. Varda Kalidas Naik Ekoskar, 2016. wireless communication using hc-05 bluetooth module. [Don Bosco College Of Engineering] 